\title{
ON THE INCOMPATIBILITY BETWEEN TWO AIFS*
}

\author{
E. CASTIÑNEA ${ }^{\dagger}$ and S. CUBILLO \\ Dept. Mat. Aplicada, Fac. de Informática, Universidad Politécnica de Madrid \\ 28660 Boadilla del Monte, Madrid \\ W. MONTILLA \\ Área de Matemática, Universidad Nacional Abierta \\ Centro Local Barinas, 5201 Venezuela \\ E-mail: willmer-montilla@hotmail.com
}

The purpose of this paper is to commence studying the incompatibility in the Atanassov's intuitionistic fuzzy sets framework. In order to do this, firstly we deal with the concept of $\mathcal{T}$-incompatible sets, where $\mathcal{T}$ is an intuitionistic tnorm, relating it with the $\mathcal{N}$-contradictory sets, where $\mathcal{N}$ is a intuitionistic fuzzy negation.

Next, an axiomatic model for measuring $\mathcal{T}$-incompatibility is introduced, and finally some methods for obtaining families of such measures are provided.

Keywords: Atanassov's intuitionistic fuzzy sets, intuitionistic t-norm, trepresentable t-norm, negations, incompatibility, contradiction.

\section{Introduction}

In the classic set theory, it is said two sets are incompatible if its intersection is the empty set; moreover, two sets $A$ and $B$ are incompatible if and only if $A$ is contained in the complementary set of $B, A \subset B^{c}$, that is, $A$ and $B$ are contradictory. Nevertheless, if these concepts, incompatibility and contradiction, are translated to the fuzzy set theory then they are not equivalent. $\mathrm{In}^{1}$, the study on the incompatibility is tackled and an axiomatic model for measuring the incompatibility between two fuzzy sets is introduced. The purposes of this work are: to establish a mathematical model for studying the incompatibility property in the Atanassov's intuitionistic fuzzy sets framework relating it to the contradiction; and to formulate the conditions a function must satisfy for measuring such property.

* This paper is partially supported by CICYT (Spain) under Project TIN 2005-08943C02-01.

$\dagger^{\dagger}$ Corresponding author 


\section{Preliminaries}

As it is well known, an Atanassov' intuitionistic fuzzy set (AIFS) on a universe $E$ is a set $A=\left\{\left(x, \mu_{A}(x), \nu_{A}(x)\right) \mid x \in E\right\}$, where $\mu_{A}, \nu_{A}: E \rightarrow[0,1]$ satisfy that $\mu_{A}(x)+\nu_{A}(x) \leq 1$ for all $x \in E$ (see $\left.{ }^{2}\right)$. Hence, $A$ is an $\mathbb{L}$-fuzzy set $\left(\mathrm{see}^{3}\right)$, where $\mathbb{L}=\left\{\bar{a}=\left(a_{1}, a_{2}\right) \in[0,1]^{2} \mid a_{1}+a_{2} \leq 1\right\}$ is a bounded and complete lattice with the order defined by: $\left(a_{1}, a_{2}\right) \leq_{\mathbb{L}}\left(b_{1}, b_{2}\right)$ if and only if $a_{1} \leq b_{1}$ and $a_{2} \geq b_{2}$, which greatest element is $1_{\mathbb{L}}=(1,0)$ and the lowest is $0_{\mathbb{L}}=(0,1)$. Thus $\chi^{A}=\left(\mu_{A}, \nu_{A}\right) \in \mathbb{L}^{E}$ is the $\mathbb{L}$-membership function of $A$.

The notions of incompatibility and contradiction in classical set theory are straight spread to the AIFS framework; and in order to do these extensions, it is necessary to have operations allowing to model the intersection and the negation; so to turn to the notion of t-norm is necessary.

Definition $2.1\left({ }^{4}\right)$. An intuitionistic t-norm is a binary operation $\mathcal{T}$ : $\mathbb{L}^{2} \rightarrow \mathbb{L}$ satisfying the following properties: commutativity, associativity, monotonicity (if $\bar{a}, \bar{b}, \bar{c} \in \mathbb{L}$ s.t. $\bar{a} \leq_{\mathbb{L}} \bar{b}$, then $\mathcal{T}(\bar{a}, \bar{c}) \leq \mathcal{T}(\bar{b}, \bar{c})$ ), and $1_{\mathbb{L}}$ is the neutral element $\left(\mathcal{T}\left(\bar{a}, 1_{\mathbb{L}}\right)=\bar{a}, \forall \bar{a} \in \mathbb{L}\right)$.

Moreover, if there exist a t-norm $T$ and a t-conorm $S$ (that is, $T$ and $S$ are binary operations on $[0,1]$ that are commutative, associative, monotonic on the both places, and with neutral element, 1 and 0 respectively; see ${ }^{5}$ ) such that $\mathcal{T}(\bar{a}, \bar{b})=\left(T\left(a_{1}, b_{1}\right), S\left(a_{2}, b_{2}\right)\right)$ for all $\bar{a}=\left(a_{1}, a_{2}\right), \bar{b}=\left(b_{1}, b_{2}\right)$, then $\mathcal{T}$ is said to be t-representable ${ }^{4}$, and it will be denoted by $\mathcal{T}_{T S}$.

Definition $2.2\left({ }^{6}\right)$. A function $\mathcal{N}: \mathbb{L} \rightarrow \mathbb{L}$ is an intuitionistic fuzzy negation (IFN) if it is decreasing and satisfies $\mathcal{N}\left(0_{\mathbb{L}}\right)=1_{\mathbb{L}}$ and $\mathcal{N}\left(1_{\mathbb{L}}\right)=0_{\mathbb{L}}$. Moreover, $\mathcal{N}$ is said to be strong (S-IFN) if it is involutive, that is, $\mathcal{N}(\mathcal{N}(\bar{a}))=\bar{a}$ for all $\bar{a} \in \mathbb{L}$.

Any S-IFN $\mathcal{N}$ is characterized by a strong negation $N$ (that is, $N$ : $[0,1] \rightarrow[0,1]$ is a decreasing and involutive function such that $N(0)=1$ and $N(1)=0)$ by means of the formula $\mathcal{N}\left(a_{1}, a_{2}\right)=\left(N\left(1-a_{2}\right), 1-N\left(a_{1}\right)\right)$, for all $\left(a_{1}, a_{2}\right) \in \mathbb{L}\left(\operatorname{see}^{6}\right.$ and $\left.^{7}\right)$. If $N=1-i d$, then the S-IFN associated with $N$ is $\mathcal{N}_{s}\left(a_{1}, a_{2}\right)=\left(a_{2}, a_{1}\right)$ and it is called standard IFN.

\section{Incompatibility and contradiction between AIFS}

The notion of contradictory AIFS was introduced in ${ }^{8}$ while that of incompatible AIFS in ${ }^{9}$. In this section, we will deepen in these concepts and their relations.

Definition 3.1 $\left({ }^{9}\right)$. Let $\mathcal{T}: \mathbb{L}^{2} \rightarrow \mathbb{L}$ be an intuitionistic t-norm. The AIFS $A$ and $B$, or alternatively $\chi^{A}$ and $\chi^{B}$, are $\mathcal{T}$-incompatible if $\mathcal{T}\left(\chi^{A}(x), \chi^{B}(x)\right)=0_{\mathbb{E}}$ for all $x \in E$. 
Now let us introduce a more general definition about the contradiction concept than definition set out in ${ }^{8}$ since it does not demand the involutive property of the negation.

Definition 3.2. Let $\chi^{A}, \chi^{B} \in \mathbb{L}^{E}$ and $\mathcal{N}$ an IFN, we say that $\chi^{A}$ is $\mathcal{N}$ contradictory with $\chi^{B}$ if $\chi^{A} \leq_{\mathbb{L}} \mathcal{N} \circ \chi^{B}$. If $\chi^{A}$ is $\mathcal{N}$-contradictory with $\chi^{B}$ and $\chi^{B}$ is $\mathcal{N}$-contradictory with $\chi^{A}$, we say that $\chi^{A}$ and $\chi^{B}$ are $\mathcal{N}$ contradictory.

Example 3.1. Let $E$ be any non-empty set and $\mathcal{N}$ the negation defined for each $\bar{a}=\left(a_{1}, a_{2}\right) \in \mathbb{L}$ by $\mathcal{N}(\bar{a})=\left(a_{2}^{2}, a_{1}^{2}\right)$. If for all $x \in E$, $\chi^{A}(x)=(0,1 / 4)$ and $\chi^{B}(x)=(1 / 2,1 / 2)$, then for all $x \in E, \chi^{A}(x) \leq_{\mathbb{L}}$ $\mathcal{N}\left(\chi^{B}(x)\right)=(1 / 4,1 / 4)$ and $\chi^{B}(x) \not \mathbb{L}_{\mathbb{L}} \mathcal{N}\left(\chi^{A}(x)\right)=(1 / 16,0)$. Hence, $\chi^{A}$ is $\mathcal{N}$-contradictory with $\chi^{B}$, but $\chi^{B}$ is not $\mathcal{N}$-contradictory with $\chi^{A}$. $\triangleleft$

In the particular case in which $\mathcal{N}$ is a strong IFN, the previous definition is the same that given in ${ }^{8}$.

In the following, let us illustrate some cases in which incompatibility and contradiction on AIFS coincide and another ones in which they behave as different concepts. For this, we need to set out a previous result about negations constructed through t-norms.

Proposition 3.1. Let $\mathcal{T}$ be a continuous intuitionistic t-norm, then the function $\mathcal{N}_{\mathcal{T}}: \mathbb{L} \rightarrow \mathbb{L}$ defined for each $\bar{a} \in \mathbb{L}$ by $\mathcal{N}_{\mathcal{T}}(\bar{a})=$ $\operatorname{Sup}\left\{\bar{b} \in \mathbb{L} \mid \mathcal{T}(\bar{a}, \bar{b})=0_{\mathbb{L}}\right\}$ is an intuitionistic fuzzy negation.

Proof. As $0_{\mathbb{L}}$ is the absorbent element of $\mathcal{T}$ (i.e. $\mathcal{T}\left(\bar{a}, 0_{\mathbb{L}}\right)=0_{\mathbb{L}}, \forall \bar{a} \in \mathbb{L}$ ), then $\mathcal{N}_{\mathcal{T}}\left(0_{\mathbb{L}}\right)=1_{\mathbb{L}}$; and as $1_{\mathbb{L}}$ is the neutral element, $\mathcal{N}_{\mathcal{T}}\left(1_{\mathbb{L}}\right)=0_{\mathbb{L}}$ is followed. Finally, taking into account that $\mathcal{T}$ is an increasing function, if $\bar{a}, \bar{b} \in \mathbb{L}$ satisfy $\bar{a} \leq_{\mathbb{L}} \bar{b}$, then $\mathcal{N}_{\mathcal{T}}(\bar{b}) \leq_{\mathbb{L}} \mathcal{N}_{\mathcal{T}}(\bar{a})$.

Proposition 3.2. Let $\mathcal{T}$ be a continuous intuitionistic t-norm and $\mathcal{N}_{\mathcal{T}}$ its associated negation. If $\chi^{A}, \chi^{B} \in \mathbb{L}^{E}$ the following is satisfied: $\chi^{A}, \chi^{B}$ are $\mathcal{T}$-incompatible if and only if $\chi^{A}, \chi^{B}$ are $\mathcal{N}_{\mathcal{T}}$-contradictory.

Proof. Notice that $\mathcal{N}_{\mathcal{T}}\left(\chi^{B}(x)\right)=\operatorname{Sup}\left\{\bar{b} \in \mathbb{L} \mid \mathcal{T}\left(\chi^{B}(x), \bar{b}\right)=0_{\mathbb{L}}\right\}$ for all $x \in E$. Thus, from $\mathcal{T}\left(\chi^{B}(x), \chi^{A}(x)\right)=0_{\mathbb{L}}$ it follows that, for all $x \in E$, $\chi^{A}(x) \leq_{\mathbb{L}} \mathcal{N}_{\mathcal{T}}\left(\chi^{B}(x)\right)$, that is, $\chi^{A}$ is $\mathcal{N}_{\mathcal{T}}$-contradictory with $\chi^{B}$; moreover having into account that $\mathcal{T}$ is commutative, it is obtained that $\chi^{B}$ is $\mathcal{N}_{\mathcal{T}}$-contradictory with $\chi^{A}$, therefore $\chi^{A}, \chi^{B}$ are $\mathcal{N}_{\mathcal{T}}$-contradictory. Reciprocally, if $\chi^{A} \leq_{\mathbb{L}} \mathcal{N}_{\mathcal{T}} \circ \chi^{B}$, due to $\mathcal{T}$ is an increasing function and to the definition of $\mathcal{N}_{\mathcal{T}}\left(\chi^{B}(x)\right)$, we have that $\mathcal{T}\left(\chi^{B}(x), \chi^{A}(x)\right) \leq_{\mathbb{L}}$ $\mathcal{T}\left(\chi^{B}(x), \mathcal{N}_{\mathcal{T}}\left(\chi^{B}(x)\right)\right)=0_{\mathbb{L}}$ for all $x \in E$. 
A particular case of this proposition is significant because the negation $\mathcal{N}_{\mathcal{T}}$ that is associated with the t-norm is an S-IFN, and in general, $\mathcal{N}_{\mathcal{T}}$ does not satisfy involutive property. Before establishing this case, to recall some concepts is opportune: If $W, W^{*}$ symbolize the Eukasiewicz's t-norm and $t$-conorm, respectively (i.e. $W(a, b)=\operatorname{Max}(0, a+b-1)$ and $W^{*}(a, b)=$ $\operatorname{Min}(a+b, 1)$ for all $a, b \in[0,1])$, then $W_{\varphi}=\varphi^{-1} \circ W \circ(\varphi \times \varphi)$ and $W_{\varphi}^{*}=\varphi^{-1} \circ W^{*} \circ(\varphi \times \varphi)$ are the t-norm and t-conorm conjugated to $W$ and $W^{*}$ by means of the order automorphism of the unit interval $\varphi$ (a bijective and strictly increasing function on $[0,1])$.

Corollary 3.1. Let $\varphi$ be an order automorphism of the unit interval, $W_{\varphi}, W_{\varphi}^{*}$ the conjugated Eukasiewicz's t-norm and t-conorm by means of $\varphi$, and $\mathcal{T}_{W_{\varphi} W_{\varphi}^{*}}$ the t-representable t-norm associated with $W_{\varphi}$ and $W_{\varphi}^{*}$. Given

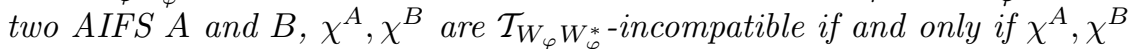
are $\mathcal{N}_{\varphi}$-contradictory, where $\mathcal{N}_{\varphi}$ is the $S$-IFN defined for each $\left(a_{1}, a_{2}\right) \in \mathbb{L}$ by $\mathcal{N}_{\varphi}\left(a_{1}, a_{2}\right)=\left(\varphi^{-1}\left(1-\varphi\left(a_{1}\right)\right), \varphi^{-1}\left(1-\varphi\left(a_{2}\right)\right)\right.$.

Example 3.2. Let $E \neq \emptyset$, if $\varphi(a)=a^{2}$ for all $a \in[0,1]$, then $\chi^{A}=$ $\left(\mu_{A}, \nu_{A}\right), \chi^{B}=\left(\mu_{B}, \nu_{B}\right) \in \mathbb{L}^{E}$ are $\mathcal{T}_{W_{\varphi} W_{\varphi}^{*}}$-incompatible if and only if $\mu_{A}(x)^{2}+\mu_{B}(x)^{2} \leq 1$ and $\nu_{A}(x)^{2}+\nu_{B}(x)^{2} \geq 1$. Thus the sets defined for all $x \in E$ by $\chi^{A}(x)=(3 / 4,0)$ and $\chi^{B}(x)=(1 / 2,1 / 2)$ are neither $\mathcal{T}_{W_{\varphi} W_{\varphi}^{*-}}$ incompatible nor $\mathcal{N}_{\varphi}$-contradictory; whereas the sets defined for all $x \in E$ by $\chi^{C}(x)=(0,1 / \sqrt{2})$ and $\chi^{D}(x)=(1 / 4,3 / 4)$ are $\mathcal{T}_{W_{\varphi} W_{\varphi}^{*} \text {-incompatible as }}$ well as $\mathcal{N}_{\varphi}$-contradictory.

\section{Incompatibility measures on AIFS}

Taking into account that not all the incompatible sets have the same behavior, that is, some of them could become compatible with small variations while others remain incompatible even considering great disturbances, it seems suitable for measuring how far two AIFS are to be compatible.

Definition 4.1. Given an intuitionistic t-norm $\mathcal{T}$, the function $I_{\mathcal{T}}: \mathbb{L}^{E} \times$ $\mathbb{L}^{E} \rightarrow[0,1]$ is said to be a $\mathcal{T}$-incompatibility measure if it satisfies:

(i) $I_{\mathcal{T}}\left(\chi^{0_{\mathbb{L}}}, \chi^{0_{\mathbb{L}}}\right)=1$.

(ii) Given $\chi^{A}, \chi^{B} \in \mathbb{L}^{E}$, if there exists $x \in E$ such that $\mathcal{T}\left(\chi^{A}(x), \chi^{B}(x)\right) \neq$ $0_{\mathbb{L}}$, then $I_{\mathcal{T}}\left(\chi^{A}, \chi^{B}\right)=0$.

(iii) Symmetry: For all $\chi^{A}, \chi^{B} \in \mathbb{L}^{E}, I_{\mathcal{T}}\left(\chi^{A}, \chi^{B}\right)=I_{\mathcal{T}}\left(\chi^{B}, \chi^{A}\right)$.

(iv) Antimonotonicity: Given $\chi^{A}, \chi^{B} \in \mathbb{L}^{E}$ such that $\chi^{A} \leq_{\mathbb{L}} \chi^{B}$, then $I_{\mathcal{T}}\left(\chi^{A}, \chi^{C}\right) \geq I_{\mathcal{T}}\left(\chi^{B}, \chi^{C}\right)$ for all $\chi^{C} \in \mathbb{L}^{E}$.

The following two results, which proofs are omitted due to the limits of space, provide some methods to construct incompatibility measures. 
Proposition 4.1. For any intuitionistic t-norm $\mathcal{T}, I_{\mathcal{T}}: \mathbb{L}^{E} \times \mathbb{L}^{E} \rightarrow[0,1]$ defined by

$$
I_{\mathcal{T}}\left(\chi^{A}, \chi^{B}\right)= \begin{cases}0, \quad \text { if } \exists x \in E, \mathcal{T}\left(\chi^{A}(x), \chi^{B}(x)\right) \neq 0 & 0_{\mathbb{L}} \\ \frac{\operatorname{Min}\left(d\left(\chi^{A}(X), 1_{\mathbb{L}}\right), d\left(\chi^{B}(X), 1_{\mathbb{L}}\right)\right)}{\sqrt{2}}, & \text { otherwise }\end{cases}
$$

where $d$ is the Euclidean distance, is a $\mathcal{T}$-incompatibility measure.

Let us construct a family of incompatibility functions that are associated with the family of order automorphisms of the unit interval. For each automorphism $\varphi$, let $\mathcal{T}_{W_{\varphi} W_{\varphi}^{*}}$ be the t-representable t-norm as it has been previously defined, then $\chi^{A^{\varphi}}=\left(\mu_{A}, \nu_{A}\right), \chi^{B}=\left(\mu_{B}, \nu_{B}\right) \in \mathbb{L}^{E}$ are $\mathcal{T}_{W_{\varphi} W_{\varphi}^{*-}}$ incompatible if and only if, for all $x \in E, \varphi\left(\mu_{A}(x)\right)+\varphi\left(\mu_{B}(x)\right) \leq 1$ and $\varphi\left(\nu_{A}(x)\right)+\varphi\left(\nu_{B}(x)\right) \geq 1$. Thus, the sets

$$
\begin{aligned}
& R_{\varphi}=\left\{(a, b) \in[0,1]^{2} \mid \varphi(a)+\varphi(b) \leq 1\right\} \\
& R_{\varphi}^{*}=\left\{(a, b) \in[0,1]^{2} \mid \varphi(a)+\varphi(b) \geq 1\right\}
\end{aligned}
$$

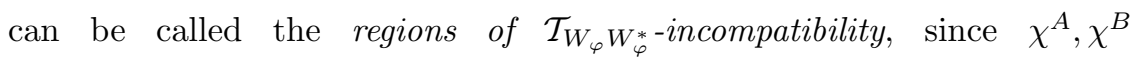

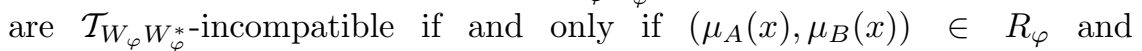
$\left(\nu_{A}(x), \nu_{B}(x)\right) \in R_{\varphi}^{*}$ for all $x \in E$ (see Fig. 1). Now we can establish the following result in which the distances to the $\mathcal{T}_{W_{\varphi} W_{\varphi}^{*}}$-incompatibility regions are considered for measuring the incompatibility.

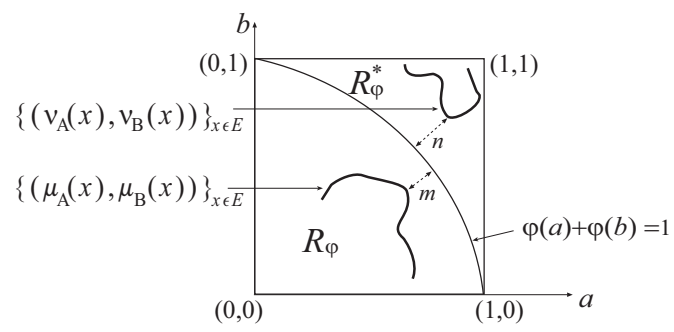

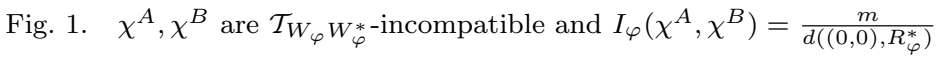

Proposition 4.2. Let $\varphi$ be an order automorphism of $[0,1]$ and $W_{\varphi}, W_{\varphi}^{*}$ be the conjugated Lukasiewicz's t-norm and t-conorm by means of $\varphi$. If $\mathcal{T}_{W_{\varphi} W_{\varphi}^{*}}$ is the t-representable t-norm associated with $W_{\varphi}$ and $W_{\varphi}^{*}$, consider $I_{\varphi}: \mathbb{L}^{E} \times \mathbb{L}^{E} \rightarrow[0,1]$ such that, for each $\chi^{A}=\left(\mu_{A}, \nu_{A}\right), \chi^{B}=\left(\mu_{B}, \nu_{B}\right) \in \mathbb{L}^{E}$, $I_{\varphi}\left(\chi^{A}, \chi^{B}\right)=\operatorname{Min}\left(\frac{\operatorname{Inf}_{x \in E} d\left(\left(\mu_{A}(x), \mu_{B}(x)\right), R_{\varphi}^{*}\right)}{d\left((0,0), R_{\varphi}^{*}\right)}, \frac{\operatorname{Inf}_{x \in E} d\left(\left(\nu_{A}(x), \nu_{B}(x)\right), R_{\varphi}\right)}{d\left((1,1), R_{\varphi}\right)}\right)$, where $d$ is the Euclidean distance, and $R_{\varphi}$ and $R_{\varphi}^{*}$ are the $\mathcal{T}_{W_{\varphi} W_{\varphi}^{*-}}$ incompatibility regions defined in equation (1). Then, $I_{\varphi}$ is a $\mathcal{T}_{W_{\varphi} W_{\varphi}^{*-}}$ incompatibility measure. 
Example 4.1. Let $E \neq \emptyset$ and $\varphi(a)=a^{2}$ for all $a \in[0,1]$. If for all $x \in E$, $\chi^{A}(x)=(0,1)$ and $\chi^{B}(x)=(1 / 2,1 / 2)$, then $\chi^{A}$ and $\chi^{B}$ are $\mathcal{T}$-incompatible for all t-norm $\mathcal{T}$. Thus, for each $\mathcal{T}$ the $\mathcal{T}$-incompatibility measure by means of the function in Proposition 4.1 is $I_{\mathcal{T}}\left(\chi^{A}, \chi^{B}\right)=1 / 2$. Whereas the $\mathcal{T}_{W_{\varphi} W_{\varphi}^{*-}}$ incompatibility measure of $\chi^{A}$ and $\chi^{B}$, by means of the function in Proposition 4.2 , is $I_{\varphi}\left(\chi^{A}, \chi^{B}\right)=\operatorname{Min}\left(\frac{1}{2}, \frac{\sqrt{5} / 2-1}{\sqrt{2}-1}\right)=\frac{\sqrt{5}-2}{2 \sqrt{2}-2}$.

\section{Conclusions}

This work is devoted to studying the incompatibility of AIFSs relating this concept with the contradiction one. For this, a generalization of the contradiction notion given in ${ }^{8}$ has been introduced, in order to get a broad framework; and a criterion for knowing when both concepts are equivalent has been proved. Since not every incompatible set behaves same, this conduct must be distinguished. Thus, an axiomatic definition for measuring the incompatibility has been presented, as well as two methods providing incompatibility measures have been set out.

\section{References}

1. E. Castiñeira, S. Cubillo and P. L. Fernández, Medidas de incompatibilidad entre conjuntos borrosos, in Actas del XIII Congreso Español sobre Tecnologías y Lógica Fuzzy (ESTYLF), (Ciudad Real (Spain), 2006).

2. K. T. Atanassov, Intuitionistic Fuzzy Sets: Theory and Applications (PhysicaVerlag, Heidelberg, 1999).

3. J. A. Goguen. L-Fuzzy Sets, Journal of Mathematical Analysis and Applications. 18-1, pp. 623-668 (1967).

4. G. Deschrijver, C. Cornelis, E. Kerre. On the representation of intuitionistic fuzzy T-norms and T-conorms, IEEE Transactions on Fuzzy Systems. 121, pp. 45-61 (2004).

5. E. P. Klement, R. Mesiar and E. Pap, Triangular Norms (Kluwer Academic Publishers, 2000).

6. H. Bustince, J. Kacprzyk and V. Mohedano. Intuitionistic Fuzzy Generators Application to Intuitionistic Fuzzy Complementation, Fuzzy Sets and Systems. 114, pp. 485-504 (2000).

7. G. Deschrijver, C. Cornelis and E. Kerre, Intuitionistic fuzzy connectives revisited, in Proc. of IX Conference of Information Processing and Management of Uncertainty in Knowledge-Based Systems (IPMU), (Annecy (France), 2002).

8. S. Cubillo and E. Castiñeira, Contradiction in intuitionistic fuzzy sets, in Proceedings IPMU, (Perugia (Italy), 2004).

9. E. Castiñeira and S. Cubillo, Algunas propiedades algebraicas de los conjuntos borrosos intuicionistas, in Actas del XII Congreso Español sobre Tecnologías y Lgica Fuzzy (ESTYLF), (Jaén (Spain), 2004). 